\title{
Effects of atomic hydrogen and deuterium exposure on high polarization GaAs photocathodes
}

\author{
M. Baylac, ${ }^{*}$ P. Adderley, J. Brittian, J. Clark, T. Day, J. Grames, J. Hansknecht, M. Poelker, M. Stutzman, and A. T. Wu \\ Thomas Jefferson National Accelerator Facility, 12000 Jefferson Avenue, Newport News, Virginia 23606, USA
}

\section{A. S. Terekhov}

Institute of Semiconductor Physics, Novosibirsk, 630090 Russia

(Received 18 September 2005; published 19 December 2005)

\begin{abstract}
Strained-layer GaAs and strained-superlattice GaAs photocathodes are used at Jefferson Laboratory to create high average current beams of highly spin-polarized electrons. High electron yield, or quantum efficiency (QE), is obtained only when the photocathode surface is atomically clean. For years, exposure to atomic hydrogen or deuterium has been the photocathode cleaning technique employed at Jefferson Laboratory. This work demonstrates that atomic hydrogen cleaning is not necessary when precautions are taken to ensure that clean photocathode material from the vendor is not inadvertently dirtied while samples are prepared for installation inside photoemission guns. Moreover, this work demonstrates that QE and beam polarization can be significantly reduced when clean high-polarization photocathode material is exposed to atomic hydrogen from an rf dissociator-style atomic hydrogen source. Surface analysis provides some insight into the mechanisms that degrade QE and polarization due to atomic hydrogen cleaning.
\end{abstract}

DOI: 10.1103/PhysRevSTAB.8.123501

PACS numbers: 29.25.Bx, 73.50.Pz, 73.61.Ey, 85.60.Ha

\section{INTRODUCTION}

The Continuous Electron Beam Accelerator Facility (CEBAF) at Jefferson Laboratory is a nuclear physics research facility where high average current beams of highly spin-polarized electrons can be delivered to three experimental end stations simultaneously (combined beam current up to $200 \mu \mathrm{A}$ with polarization $>70 \%$ ). The beams are produced by photoemission from GaAs photocathodes within ultrahigh vacuum chambers. The photocathode is formed when cesium $(\mathrm{Cs})$ and nitrogen trifluoride $\left(\mathrm{NF}_{3}\right)$ gas are applied to the surface to create a negative electron affinity condition (NEA) [1]. High electron yield, or quantum efficiency $(\mathrm{QE})$, is obtained only when the photocathode surface is atomically clean. Years ago, wetchemical etching techniques were replaced with atomic hydrogen cleaning to obtain high QE from bulk GaAs [2]. An rf-inductive discharge dissociator apparatus was chosen as the atomic hydrogen source. In this paper, the merits of atomic hydrogen cleaning are examined. Results indicate that vendors provide sufficiently clean photocathode material to warrant atomic hydrogen cleaning superfluous. Very high QE can be obtained without implementing any form of cleaning, atomic hydrogen cleaning or other techniques, if precautions are taken to ensure clean material is not inadvertently dirtied when photocathode samples are prepared for installation within photoemission guns. Moreover, atomic hydrogen cleaning

\footnotetext{
*Corresponding author.

Present address: Laboratoire de Physique Subatomique et Cosmologie, 53 Avenue des Martyrs, F-38026 Grenoble Cedex, France.

Electronic address: baylac@1psc.in2p3.fr
}

of high-polarization photocathode material, strained-layer GaAs and strained-superlattice GaAs, can actually reduce $\mathrm{QE}$ and beam polarization.

\section{OVERVIEW}

It is worth discussing the seemingly contradictory nature of past and present statements describing the benefits and drawbacks of atomic hydrogen cleaning. Original claims by the authors regarding atomic hydrogen cleaning were very favorable [2]. These claims are still valid, namely, atomic hydrogen cleaning remains an effective technique to remove surface contamination from bulk GaAs. A noteworthy difference between past and present reports is the observation that atomic hydrogen cleaning is unwarranted. This statement stems from the realization, made over the course of years, that contamination is introduced onto the surface of the photocathode material during handling. This work, in part, serves to highlight the origins of photocathode surface contamination.

The procedure for making a photocathode at Jefferson Lab involves a number of steps. First, small samples are cut from large wafers obtained from vendors. Then the outer edges of individual samples are anodized to limit the active area of the photocathode, a step that helps prolong the operating lifetime of the photoemission gun while delivering beam at high voltage $[3,4]$. Following the anodization step, samples are indium soldered to a sample holder. Historically, samples are cleaned with atomic hydrogen inside a dedicated vacuum chamber. The photocathode is then installed inside a high voltage photoemission gun at the CEBAF photoinjector and baked at $250{ }^{\circ} \mathrm{C}$ for $30 \mathrm{~h}$. Finally, the photocathode material is heated to $540{ }^{\circ} \mathrm{C}$ or $580^{\circ} \mathrm{C}$ (depending on the photocathode material) to lib- 
erate loosely bound gas, cooled and activated in the usual way with successive application of cesium and nitrogentrifluoride [1]. Three of these steps have been identified as providing opportunity for contaminating the surface of the photocathode material; wafer cutting, edge-anodizing, and gun bakeout. Each of these steps has undergone revision (as described below) and now much of the potential for contaminating the surface of the photocathode has been eliminated.

Originally, samples were cut from large wafers using a circular-shaped cutting jig and diamond-paste slurry. The large wafer was sandwiched between glass slides using an acetone-soluble adhesive in an attempt to protect the surface of the photocathode during cutting. This process was time consuming and atomic hydrogen cleaning was always an essential follow-up step to obtain reasonable QE from the photocathode. Years ago, this cutting technique was replaced with a far simpler cleaving technique. A diamondtip scribe is now used to cleave square samples from large wafers. Aside from the diamond-tip scribe, nothing touches the surface of the photocathode material during cleaving and as a result, the photocathode surface is not contaminated.

Two variations of the edge-anodizing technique have been studied at CEBAF. Before elaborating, a brief description of the motivation for anodizing the edge of the photocathode is given. At CEBAF, only the center portion of the photocathode is intentionally illuminated with laser light. Additional electrons can be emitted from the edge of the photocathode because of stray light and spontaneous emission of light within the photocathode material itself. These edge-emitted electrons travel extreme trajectories and can strike the vacuum chamber walls producing an increased vacuum load due to electron stimulated desorption. This added vacuum load diminishes photocathode lifetime via enhanced ion back-bombardment. By anodizing the edge of the photocathode, unwanted photoemission from the edge of the photocathode is eliminated.

During the edge-anodizing process [5], it is very important to keep the center portion of the wafer free of both anodization and contamination. Originally, this was accomplished by applying a small drop of acetone-soluble adhesive to the center of the sample. Following anodization, repeated rinses in acetone were required to remove the adhesive before the sample could be installed within a photogun. More recently, the adhesive has been replaced by an o-ring sealed device used to create a weak vacuum at the center of the sample while it is immersed in the electrolytic bath. The electrolytic solution does not come into contact with the center of the sample and the use of adhesives is minimized, thereby reducing the chance of residual contamination. The present method is better than the original method however, of all the preparation steps, the edge-anodization step remains the most troublesome from a contamination point of view. Efforts are under way to implement an activation mask technique described in Ref. [4], as a means to completely eliminate the need to immerse photocathode samples in solvents.

Another improvement that has served to maintain sample cleanliness is the use of nitrogen-filled glove bags when installing or removing samples from vacuum chambers. The use of a nitrogen-filled glove bag ensures that very little water vapor enters the vacuum chamber. As a result, photogun vacuum pressure quickly recovers to $<1 \times$ $10^{-8}$ Torr within minutes of pump down and ensures that pressure remains below this value for the duration of the bakeout at $250^{\circ} \mathrm{C}$. By contrast, it was not uncommon for pressure to exceed $1 \times 10^{-6}$ Torr during photogun bakeouts when nitrogen-filled glove bags were not used. It is believed that low pressure during photogun bakeout helps to preserve surface cleanliness of the photocathode.

In summary, during the early days of the polarized beam program at CEBAF, atomic hydrogen cleaning was an essential step toward obtaining high QE. However, as preparation procedures were refined, it became apparent that large doses of atomic hydrogen were no longer required and in fact vendors have been providing very clean material. More recently, it was surprising to find that besides being superfluous, atomic hydrogen can actually reduce QE and polarization from high-polarization photocathodes. These results are presented below.

\section{PHOTOCATHODES}

The same high-polarization photocathode materials that are being used at CEBAF for the nuclear physics program have been used for this experiment: strained-layer GaAs and strained-superlattice GaAs. Strained-layer GaAs samples are obtained from Bandwidth Semiconductor [6], formerly SPIRE Corporation. This material has been used at CEBAF since 1998 and typically provides polarization $75 \%$ to $80 \%$ with $\mathrm{QE} \sim 0.15 \%$. The strained-layer GaAs photocathode is manufactured using metal organic chemical vapor deposition, or MOCVD, and consists of a $100 \mathrm{~nm}$ active layer of $p$-doped GaAs grown atop a $2.5 \mu \mathrm{m}$ layer of GaAsP, with phosphorus content of 28\% (Fig. 1). The $\mathrm{Zn}$ dopant is uniformly distributed throughout the active layer at a concentration of $1 \times 10^{18}$ atoms $/ \mathrm{cm}^{3}$. This prescription follows Stanford Linear Accelerator Center (SLAC) specifications as described in Ref. [7].

Strained-superlattice GaAs samples were purchased from SVT Associates [8] and have only recently become commercially available. The results reported here and in Ref. [9] indicate this material offers great promise, providing polarization $80 \%$ to $90 \%$ and $\mathrm{QE} \sim 1 \%$, a significant improvement over strained-layer GaAs photocathodes. The strained-superlattice photocathode is manufactured using molecular beam epitaxy, or MBE, and consists of a heavily $p$-doped $5 \mathrm{~nm} \mathrm{GaAs}$ surface layer grown atop 14 pairs of alternating layers of GaAsP and GaAs (Fig. 1). Interior GaAs layers are more modestly doped compared to 


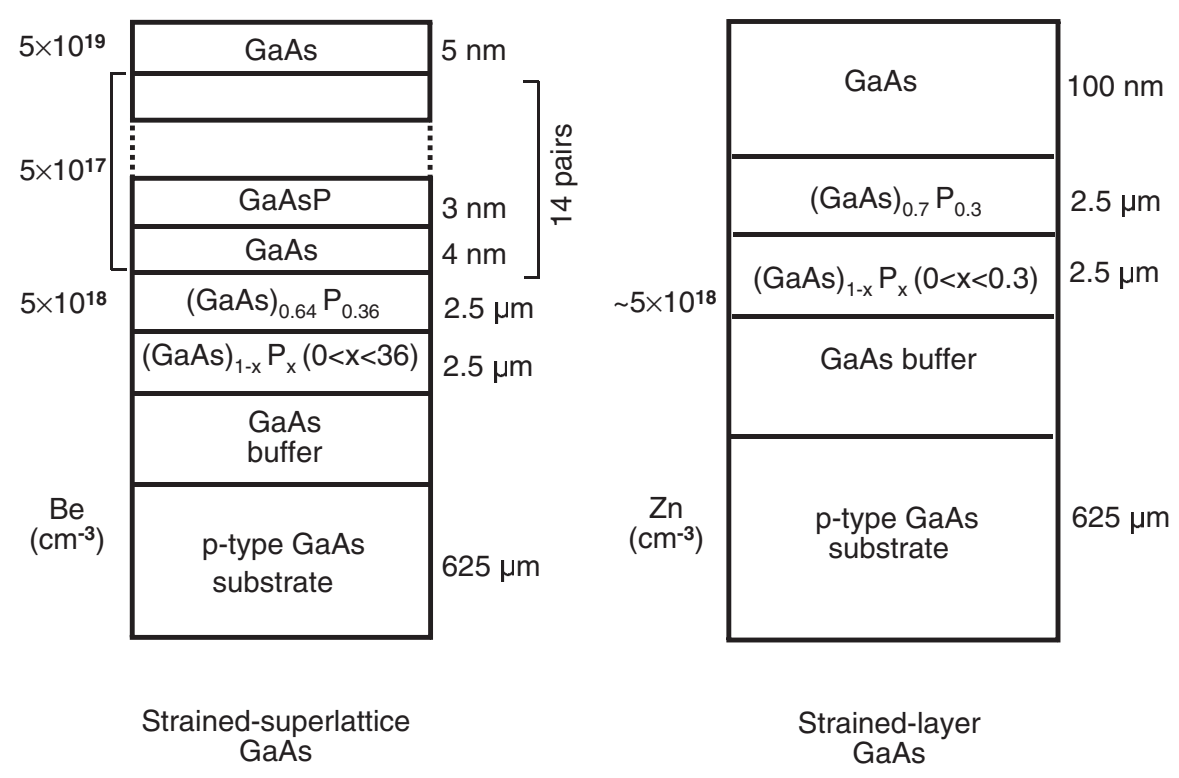

FIG. 1. Structure of the high-polarization GaAs photocathode materials studied in this paper.

the surface layer; $[\mathrm{Be}]=5 \times 10^{17}$ atoms $/ \mathrm{cm}^{3}$ versus $5 \times$ $10^{19}$ atoms $/ \mathrm{cm}^{3}$ of the surface layer. The phosphorus content of the GaAsP layers is $36 \%$. This prescription also follows SLAC specifications [9].

Throughout these tests, samples are cleaved to proper dimensions $(15.5 \mathrm{~mm} \times 15.5 \mathrm{~mm}$ or smaller) and indium soldered to standard Jefferson Lab sample holders. Samples are not treated with wet chemicals at any time throughout the experiment; no acid/base etching, degreasing, or anodization. Nitrogen-filled glove bags are always used when samples are installed into vacuum chambers to minimize contact with air and reduce the likelihood of introducing water vapor into vacuum chambers.

\section{EXPERIMENT}

Beam polarization and $\mathrm{QE}$ measurements are made using a test stand that includes a $-100 \mathrm{kV}$ photogun and a Mott polarimeter (Fig. 2). The photogun is described in Ref. [10]. It has been modified to include a simple vacuum "load-lock" section that allows the installation of new photocathode samples without baking the entire photogun
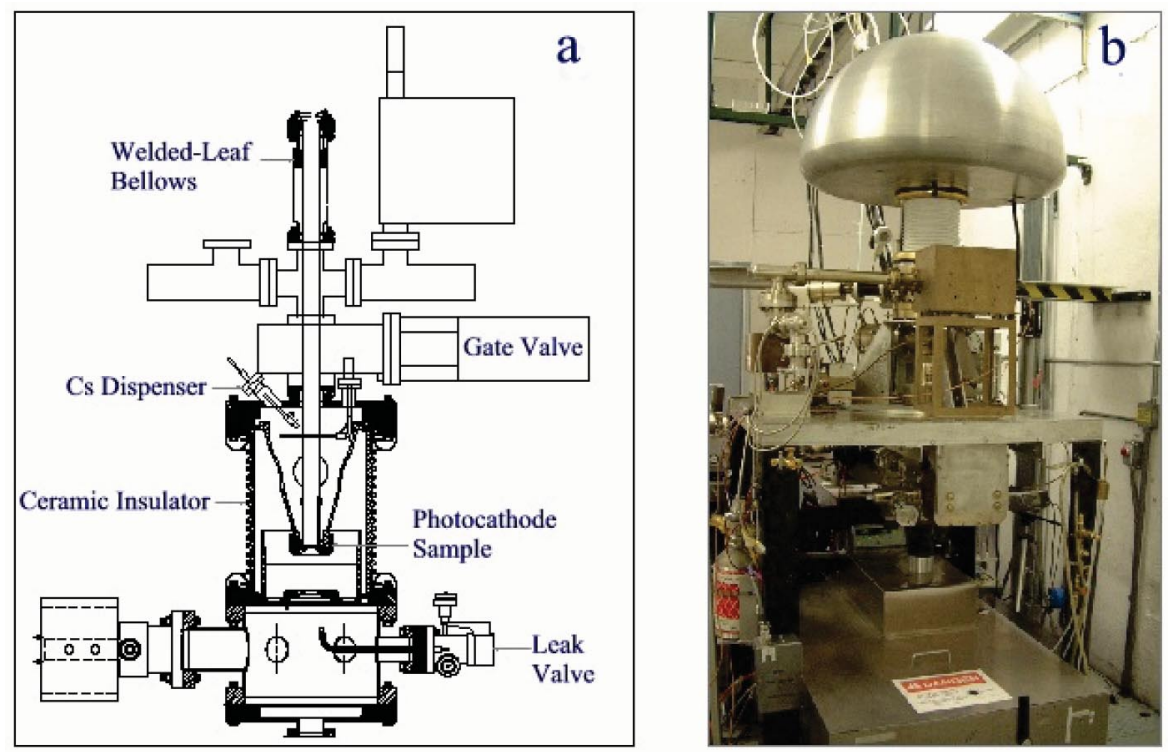

FIG. 2. (Color) (a) Schematic drawing of the $-100 \mathrm{kV}$ photogun and load-lock vacuum chamber. The load-lock vacuum chamber sits atop the photogun separated by an all-metal gate valve. Only the load-lock section of the vacuum system is baked when a sample is installed. (b) The test stand includes the photogun, beam line, and Mott polarimeter (not visible in this photograph). A remotely controlled, wavelength-tunable Ti:sapphire laser sits within the aluminum enclosure below the photogun. 
[11]. The load-lock section consists of a long welded-leaf bellows and two bakeable all-metal valves to allow pump down and vacuum isolation from the photogun. A sample holder is inserted into the bellows section of the load-lock vacuum chamber and baked at $250^{\circ} \mathrm{C}$ for $10 \mathrm{~h}$. When the bakeout is complete, the vacuum pressure in the load-lock section is $<1 \times 10^{-10}$ Torr. At this point, the valve to the photogun can be opened to allow insertion of the photocathode sample into the photogun vacuum chamber where it is heated to $540^{\circ} \mathrm{C}$ or $580^{\circ} \mathrm{C}$, depending on the photocathode material, and then cooled to room temperature prior to activation to NEA with cesium and $\mathrm{NF}_{3}$. The photocathode is activated above the cathode electrode to avoid depositing cesium on the electrode surface. Following activation, the photocathode sample is lowered into the cathode electrode. Light from a wavelengthtunable Ti:sapphire laser (Spectra Physics 3900) enters the gun chamber through a vacuum window. The laser light is tightly focused at the photocathode with $\sim 350 \mu \mathrm{m}$ diameter FWHM. The laser spot can be moved across the photocathode surface to measure QE and beam polarization across the entire sample with a high degree of precision. The laser light passes through a Pockels cell with $10 \mathrm{~Hz}$ flip rate to create left/right circularly polarized light. This produces spin-polarized electrons with spin direction parallel/antiparallel to the direction of beam travel. Electrons exit the photogun chamber and travel through a vacuum beam line to a Mott-scattering polarimeter and beam dump $\sim 5 \mathrm{~m}$ away. Solenoid magnets focus the beam to create a well-defined beam envelope and steering dipole magnets keep the beam centered throughout the apparatus. The electrons pass through an electrostatic bend to rotate the electron-spin direction orthogonal to the direction of beam propagation, a necessary condition for Mott-scattering polarimetry [12]. The electrons hit a gold foil inside the Mott polarimeter and a scattering asymmetry for the two spin states is measured using two identical silicon detectors in the horizontal plane (backscattered electrons at $\theta= \pm 120^{\circ}$ ). Measurement of the scattering asymmetry and knowledge of the analyzing power of the gold foil allows determination of beam polarization. Polarization measurements are statistically accurate to $1 \%$. The systematic uncertainty of the absolute polarization measurement is estimated to be $\sim 4 \%$ based on a cross calibration of this polarimeter against the $5 \mathrm{MeV}$ Mott polarimeter at CEBAF, which is considered to be accurate to within 1.5\% [13] and studies of the test stand polarimeter. Relative accuracy between polarization measurements of different samples is expected to be $<4 \%$, as most sources of systematic uncertainties are common to all measurements [13].

The experiment follows a sequence of steps: (a) the photocathode sample is installed into the load-locked section of the test stand, (b) the load-lock vacuum section is baked to $250^{\circ} \mathrm{C}$ for $10 \mathrm{~h}$, (c) the sample is inserted into the gun chamber and heated to $>500{ }^{\circ} \mathrm{C}$, (d) the sample is allowed to cool and then activated, (e) the sample is characterized; polarization and $\mathrm{QE}$ are measured as a function of wavelength, (f) the sample is removed from the test stand and installed on a deuterium-cleaning chamber, $(\mathrm{g})$ the sample is exposed to the rf source of atomic deuterium, (h) the sample is reinstalled into the loadlocked vacuum chamber where the process repeats.

A heat cycle always precedes photocathode activation. Heating liberates loosely bound gas at the surface and removes deuterium gas that has diffused into the material during the deuterium-cleaning step (g) above. Slightly different heating protocols are used for the two different photocathode materials prior to activation. Strained-layer GaAs photocathode samples are heated to $\sim 580^{\circ} \mathrm{C}$ for $2 \mathrm{~h}$ prior to activation. Strained-superlattice samples are heated to $\sim 540^{\circ} \mathrm{C}$ for $2 \mathrm{~h}$, slightly cooler than the strained-layer GaAs samples, to minimize diffusion of the Be dopant from the highly doped surface layer [9].

Square-shaped photocathode samples are secured to sample holders using tantalum caps with circular cutouts in the center. The active area of the photocathode is either 5 or $13 \mathrm{~mm}$ diameter, depending on how much material is available at the time of the test. Typical values of $\mathrm{QE}$ and polarization from both photocathode materials are shown in Fig. 3. These results are from samples that have not been exposed to atomic hydrogen or deuterium. The variation in QE across the sample in plots 3(a) and 3(c) is an artifact of nonuniform application of cesium due to the close proximity and orientation of the cesium dispenser relative to the photocathode sample. These variations are not indicative of variations in surface cleanliness or quality.

It should be noted that in the absence of hydrogen cleaning, photocathodes can be heated and reactivated many times without QE degradation. Hydrogen is the dominant species within CEBAF photoguns. Chemical poisoning of the photocathode surface does not occur during the heat treatment which precedes photocathode activation to NEA. For example, it is common to heat and activate photocathodes within the CEBAF $100 \mathrm{kV}$ photoguns about every 3 months over the course of years without noticeable reduction in maximum QE. A strainedsuperlattice GaAs sample was heat cleaned and activated up to 5 times in a low-voltage vacuum test chamber similar to that described in Sec. VI without QE degradation. Similarly strained-superlattice samples heated and activated repeatedly in the $100 \mathrm{kV}$ test gun and more recently, in the CEBAF photoguns, did not exhibit quantum efficiency reduction in the absence of hydrogen or deuterium cleaning.

Photocathode samples were exposed to atomic deuterium inside a separate vacuum apparatus shown in Fig. 4. Atomic hydrogen can also be created with this same vacuum apparatus by merely replacing the tank of gas shown in Fig. 3(b). Deuterium gas is chosen to provide a means to 

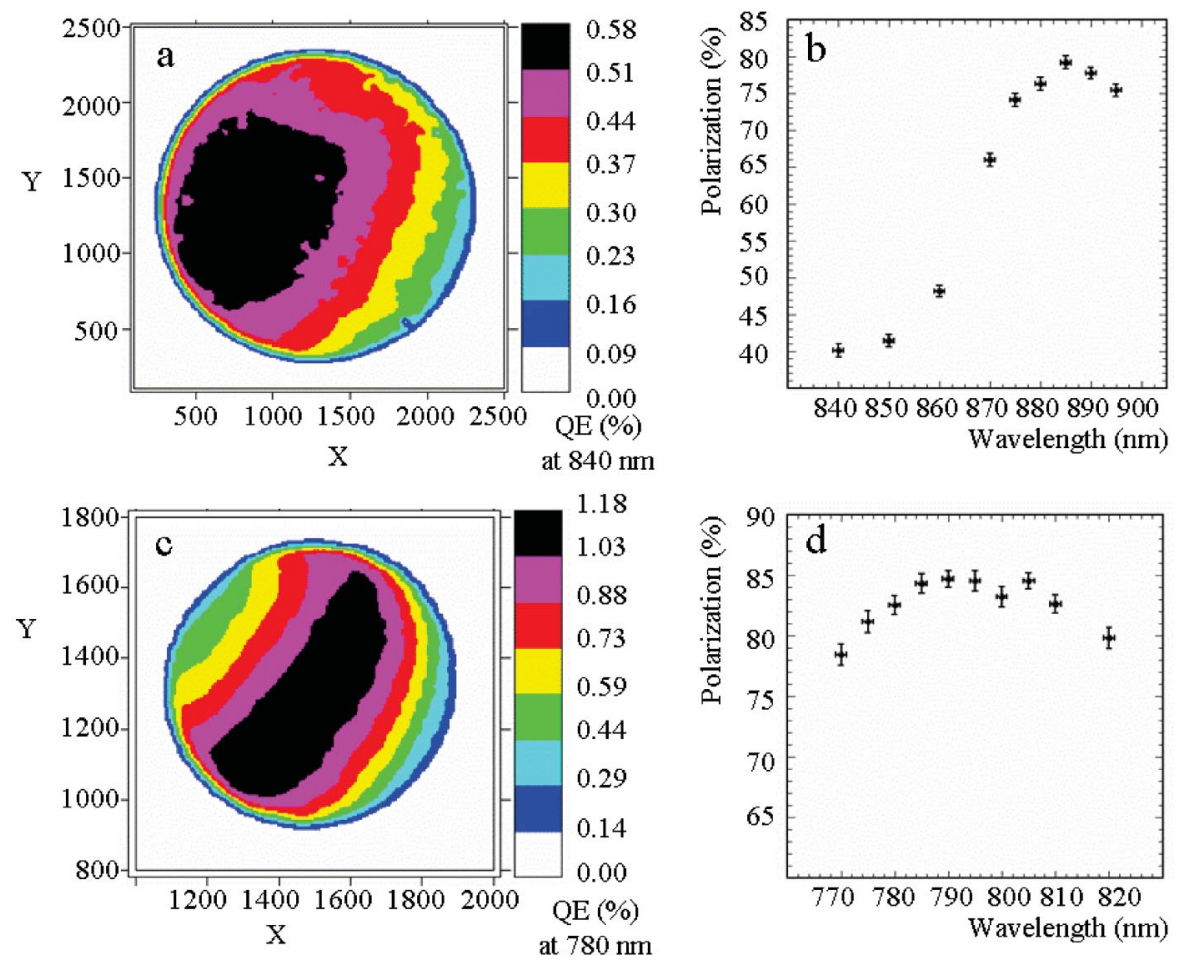

FIG. 3. (Color) QE and polarization results obtained prior to exposing samples to atomic deuterium. The top (bottom) plots show typical QE and polarization results from strained-layer GaAs (strained-superlattice GaAs). The X and Y axes in plots (a) and (c) are numbered in arbitrary stepper motor units. Polarization data in plots (b) and (d) were obtained from the central location of the photocathode, however other locations were studied to determine polarization uniformity. Only statistical errors are indicated on the plots.

help determine proper heating recipes (temperature and duration) following exposure; mass 4 can be clearly identified over the molecular hydrogen gas background as it diffuses out of the photocathode sample using a residual

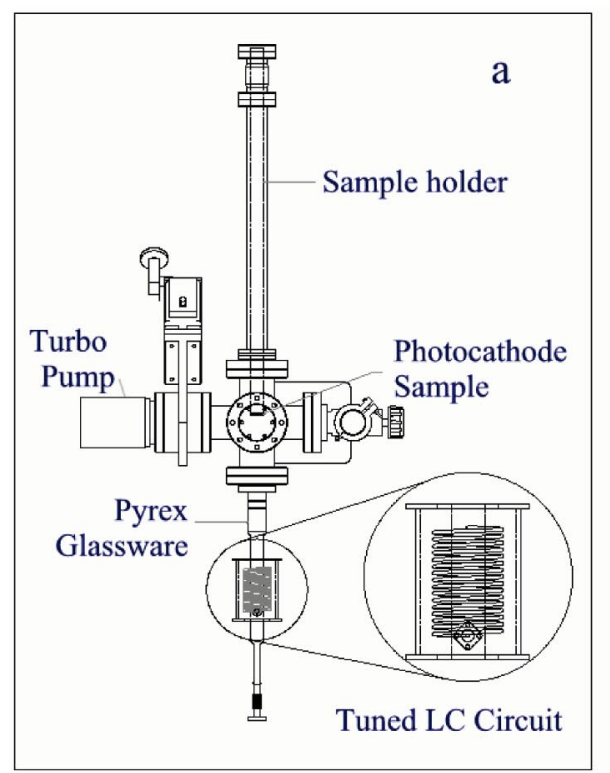

gas analyzer. Tests completed to date suggest there is no difference between the two gases with regard to the way they remove surface contamination and effect performance of high-polarization GaAs photocathodes. The deuterium

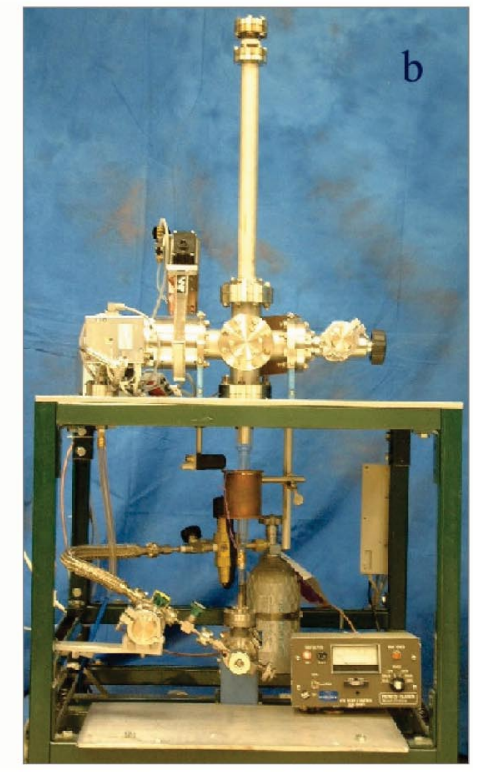

FIG. 4. (Color) The atomic hydrogen/deuterium source. Samples were installed inside this stand-alone vacuum chamber and exposed to incremental doses of atomic deuterium. A description of this vacuum chamber appears in the text. 
source used in this experiment is the same apparatus used to clean photocathode samples prior to installation within CEBAF photoguns and operating conditions are identical.

The atomic deuterium source is based on work in Ref. [14]. Commercial research-grade molecular deuterium flows through a leak valve into a Pyrex glass dissociator $(2.5 \mathrm{~cm}$ diameter). The molecular deuterium is dissociated with an rf-inductive discharge created by a 12 turn coil $3.5 \mathrm{~cm}$ in diameter, which is part of an LC tuned circuit. The LC circuit resonates at $\sim 100 \mathrm{MHz}$ and the atomic fraction is maximized when the pressure inside the dissociator is $15 \mathrm{mTorr}$ and the absorbed rf power exceeds $50 \mathrm{~W}$. Atomic deuterium exits the dissociator through a $1 \mathrm{~mm}$ diameter hole and travels through an aluminum [15] tube toward the photocathode sample approximately $15 \mathrm{~cm}$ away, which is mounted to a sample holder and located near the center of a vacuum cross. A turbomolecular pump and ion pump are used to remove the deuterium gas from the vacuum chamber. The turbomolecular pump (Balzer, $50 \mathrm{~L} / \mathrm{sec}$ ) does most of the pumping, the ion pump serves largely to monitor vacuum pressure near the sample. This pumping scheme serves to maintain pressure near the sample $\sim 1 \times 10^{-5}$ Torr, in order to provide a large mean free path for deuterium $(>1 \mathrm{~m})$. Maintaining relatively low pressure near the sample and a long mean free path are essential to ensure atomic deuterium does not recombine before hitting the sample. Monte Carlo simulations predict that approximately $2.5 \%$ of the total deuterium flux hits the photocathode sample. Under these conditions, the atomic deuterium flux at the sample is estimated around $10^{17}$ atoms $/ \mathrm{cm}^{2} / \mathrm{sec}$, assuming a $50 \%$ degree of dissociation [16].

\section{BEAM POLARIZATION}

Electron beam polarization is measured as a function of atomic deuterium exposure for multiple samples of both

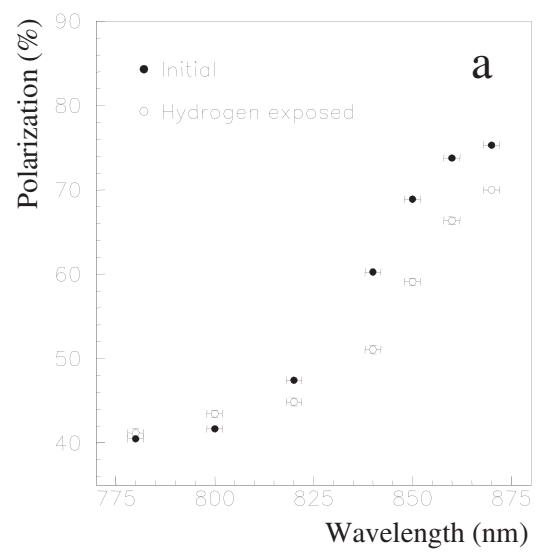

photocathode materials; strained-layer GaAs and strainedsuperlattice GaAs. Samples are characterized prior to exposure to atomic deuterium and again following repeated application of atomic deuterium. For each sample, polarization is measured as a function of laser wavelength at several locations on the photocathode. Conditions are kept constant for all measurements to minimize systematic discrepancies between data points (e.g., foil thickness, beam steering, counting rates).

Five different strained-layer GaAs samples are studied. Maximum polarization from each sample, prior to atomic deuterium exposure, is consistently between $75 \%$ and $80 \%$ with peak polarization obtained between 860 and $880 \mathrm{~nm}$. Samples are subjected to repeated doses of atomic deuterium of 15 min duration. The operating conditions of the atomic deuterium source are maintained identical with each use (e.g., sample temperature, applied and absorbed rf power, molecular deuterium flow rate). Polarization remained nearly constant for deuterium exposure up to $30 \mathrm{~min}$. However, after a cumulative exposure time of $45 \mathrm{~min}$, beam polarization decreased by an amount that exceeded the statistical and systematic error of the measurement [Fig. 5(a)] for all tested samples. The strongest depolarization is observed for wavelengths slightly lower than the wavelength that provides peak polarization. At $860 \mathrm{~nm}$, beam polarization drops from $74 \%$ to $66 \%$, an $11 \%$ reduction. Such a reduction in beam polarization would be very costly to the nuclear physics program at CEBAF since the figure of merit of most polarized beam experiments is proportional to the square of the beam polarization [17]. Beam polarization cannot be restored by merely heating and reactivating the sample. One sample is heated to $580^{\circ} \mathrm{C}$ for $12 \mathrm{~h}$ and reactivated without restoring the initial polarization.

Similar measurements are made using three different strained-superlattice GaAs samples [Fig. 5(b)]. Maximum polarization from each sample, prior to atomic deuterium

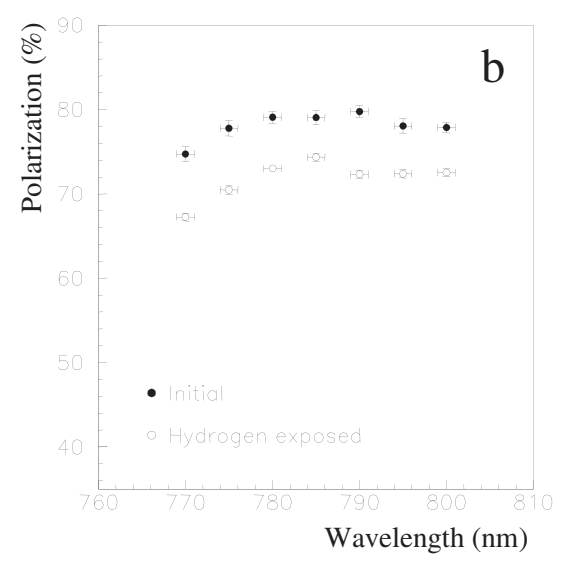

FIG. 5. Beam polarization from strained-layer GaAs (a) and strained-superlattice GaAs (b) photocathodes, before (solid dots) and after (open dots) exposure to atomic hydrogen. The strained-layer GaAs material was exposed to atomic deuterium for 45 min cumulative dose (three separate 15 min exposures). The strained-superlattice GaAs material was exposed to atomic deuterium for 30 min cumulative dose (three separate exposures of 6, 6, and $18 \mathrm{~min}$ ). Errors shown are statistical only. 
exposure, is consistently between $80 \%$ and $86 \%$ with peak polarization obtained between 775 and $795 \mathrm{~nm}$. The QE of the strained-superlattice material is more strongly adversely affected by atomic deuterium exposure, as will be described in detail below. As a result, a smaller cumulative dose is applied to the strained-superlattice GaAs material. Beam polarization drops slightly with each successive application of atomic deuterium. After $30 \mathrm{~min}$ cumulative exposure time, beam polarization is down by $\sim 10 \%$ across a broad range of wavelengths near the band gap. Merely heating and reactivating the material does not restore beam polarization.

A preliminary account of this work [11] indicated a more dramatic decrease in beam polarization following a large exposure to atomic deuterium ( $>60 \mathrm{~min}$ ). This early account was exaggerated because of unintended illumination of the photocathode with low wavelength light when attempting to measure polarization near the band gap where QE was very low. The unwanted light originated from amplified spontaneous emission (ASE) from within the Ti:sapphire laser, with most of this light centered near the wavelength $700 \mathrm{~nm}$. For work reported here, great care was taken to ensure that ASE-generated photocurrent was a negligible portion of the total beam extracted from the photocathode. This necessitated that all measurements were obtained with $\mathrm{QE} \geq 0.05 \%$ and that conditions of the optical system were kept constant. Systematic checks were done on a regular basis throughout the experiment to ensure that the observed polarization variations were genuine and not a result of systematic error associated with inadvertent illumination of the photocathode with low wavelength light.

\section{QUANTUM EFFICIENCY}

The QE of each photocathode sample is measured as a function of exposure to atomic deuterium. Quantum efficiency is defined as the ratio of extracted electrons to the number of incident photons and can be calculated (in \%) using the simple equation:

$$
\mathrm{QE}=\frac{124 I}{P \lambda},
$$

where $I$ is the photocurrent measured at the beam dump in units of $\mu \mathrm{A}, P$ is incident laser power measured outside the vacuum chamber in units of $\mathrm{mW}$, and $\lambda$ is the laser wavelength measured in units of nanometers. The QE of both photocathode materials is observed to decrease significantly with each application of atomic deuterium. For strained-layer GaAs samples, QE drops from typical maximum value of $0.2 \%$ to $\sim 0.08 \%$ after a 45 min cumulative dose of atomic deuterium. The maximum QE of strainedsuperlattice samples drops more drastically: from $1 \%$ to $0.2 \%$, after the same exposure time. Repeated heat treatments and reactivations could not restore the initial QE: the quantum efficiency remains stable in the absence of deuterium exposure, as explained in Sec. IV.
To address the concern that $\mathrm{QE}$ reduction might be a result of repeated bakeouts within the relatively poor vacuum of the heated load-lock chamber of the high voltage test stand, separate tests are conducted in a low-voltage vacuum chamber that contained its own atomic hydrogen $\mathrm{rf}$ source. Once loaded into the low-voltage test stand, a sample can be repeatedly exposed to atomic hydrogen without transferring the sample at atmospheric pressure to a different vacuum chamber and then rebaked, as is the case for the high voltage test stand. From a photogun standpoint, the low-voltage gun is very similar to the high voltage gun, having similar components except that samples are biased at $-200 \mathrm{~V}$ rather than $-100 \mathrm{kV}$. No magnets are required to deliver the extracted electron beam to a beam dump; the electrons leave the photocathode surface and travel $\sim 15 \mathrm{~cm}$ to the grounded vacuum chamber wall. Samples are loaded into this apparatus using a nitrogen-filled glove bag and the entire vacuum chamber is baked (only once) at $250{ }^{\circ} \mathrm{C}$ for $30 \mathrm{~h}$. Pressure inside the chamber is $<10^{-10}$ Torr post-bakeout. Samples are heated prior to activation following the recipe described previously.

Atomic hydrogen is chosen for measurements using this chamber to investigate possible differences between hydrogen and deuterium (none have been found). The atomic hydrogen source is located below the photocathode sample and is similar to that shown in Fig. 4 except that hydrogen is pumped from the vacuum chamber using nonevaporable getter pumps instead of a turbomolecular pump. After application of atomic hydrogen, the vacuum pressure inside the vacuum chamber is allowed to recover over night to pressure $<10^{-10}$ Torr. The pressure inside the chamber rises to $<10^{-8}$ Torr during sample heating but this gas is primarily hydrogen that diffuses from the sample and the sample holder. Photocathodes are activated the usual way, with successive application of cesium and nitrogen trifluoride. Quantum efficiency is measured throughout the activation process using diode lasers at $840 \mathrm{~nm}$ wavelength for strained-layer GaAs and at $780 \mathrm{~nm}$ wavelength for strained-superlattice GaAs. Results are shown in Fig. 6, where $\mathrm{QE}$ is plotted versus atomic hydrogen exposure time. To maintain consistency between each activation, the QE numbers in Fig. 6 correspond to 13 applications of cesium and nitrogen trifluoride. The QE of both photocathode materials drops steadily with each successive application of atomic hydrogen in a manner consistent with measurements from the vent-bake high voltage apparatus. Because similar results are obtained in both systems, the QE reduction cannot simply be attributed to repeated bakeouts in poor vacuum or to differences between hydrogen and deuterium

\section{SURFACE ANALYSIS}

Numerous publications describe the effects of atomic hydrogen on semiconductor surfaces. Among them, 

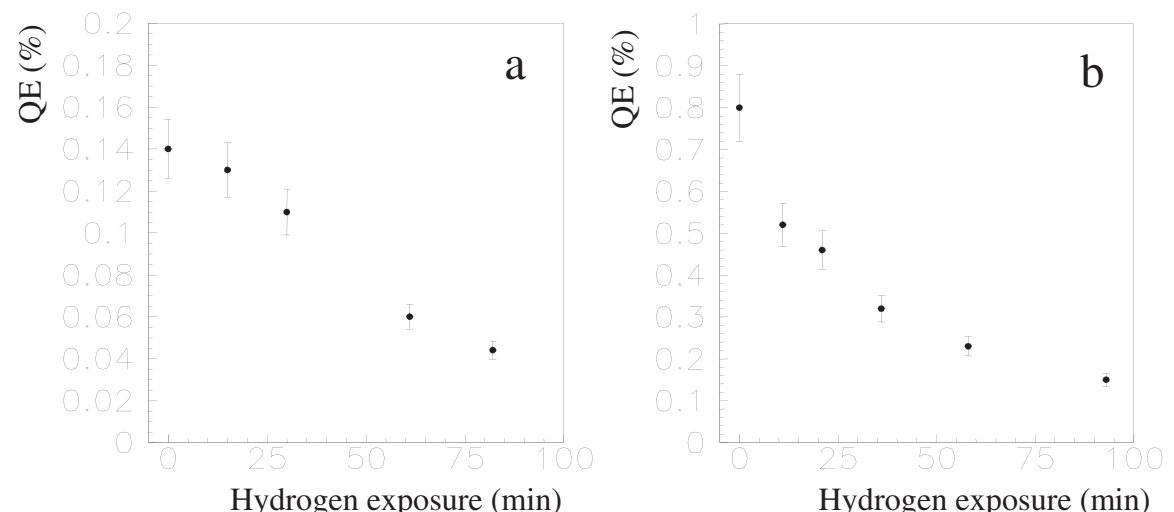

FIG. 6. Quantum efficiency versus hydrogen exposure duration for strained-layer GaAs at $840 \mathrm{~nm}$ (a) and for strained-superlattice GaAs at $780 \mathrm{~nm}(\mathrm{~b})$ in the low-voltage chamber.

Refs. [18-22] describe the mechanisms by which atomic hydrogen reacts with oxygen and carbon compounds on the surface of GaAs. Although some discrepancies exist between each reference, conclusions are generally consistent, namely, atomic hydrogen reacts with oxygen and carbon on the surface to form volatile compounds that are subsequently liberated and pumped away by the vacuum system. Simple rate equations help to appreciate the chemical reactions that liberate oxides from the surface:

$$
\begin{aligned}
& 2 \mathrm{As}_{2} \mathrm{O}_{x}+4 x \mathrm{H} \rightarrow 2 x \mathrm{H}_{2} \mathrm{O}+\mathrm{As}_{4} \\
& \text { [Eq. (2), from Ref. [18]], } \\
& \mathrm{Ga}_{2} \mathrm{O}_{3}+4 \mathrm{H} \rightarrow 2 \mathrm{H}_{2} \mathrm{O}+\mathrm{Ga}_{2} \mathrm{O} \\
& \text { [Eq. (3), from Ref. [19]], }
\end{aligned}
$$

where $x=1,2$, or 5 and represents various arsenic oxides. Arsenic oxides are efficiently removed from the surface at room temperature [18]. Higher temperatures are required to completely remove $\mathrm{Ga}$ oxides, $25^{\circ} \mathrm{C}$ to $400{ }^{\circ} \mathrm{C}$, depending on the reference. Carbon contamination is thought to be removed as a result of formation of $\mathrm{H}_{2} \mathrm{O}$ and $\mathrm{CH}_{4}$ and subsequent desorption of these compounds from the surface [21].

Many of the cited references report a potential drawback of the atomic hydrogen cleaning technique, namely, excessive exposure of GaAs samples to atomic hydrogen leads to arsenic depletion of the sample surface $[19,20]$. Arsenic depletion combined with nonuniform distribution and composition of contamination across the GaAs surface could lead to surface roughening, which in principle could affect both QE and beam polarization for the following reasons. A rough surface, being more reflective, would exhibit lower QE because less light would enter the photocathode material. In addition, the polarization would be reduced because circularly polarized laser light will appear as elliptically polarized light on a rough photocathode surface. To test this idea, a surface profilometer [23] is used to compare surface roughness of each highpolarization photocathode material and bulk GaAs, before and after exposure to atomic deuterium. Each scan probes an area of $200 \times 200 \mu \mathrm{m}^{2}$ (Fig. 7). The rms surface roughness is averaged over several locations for each exposure
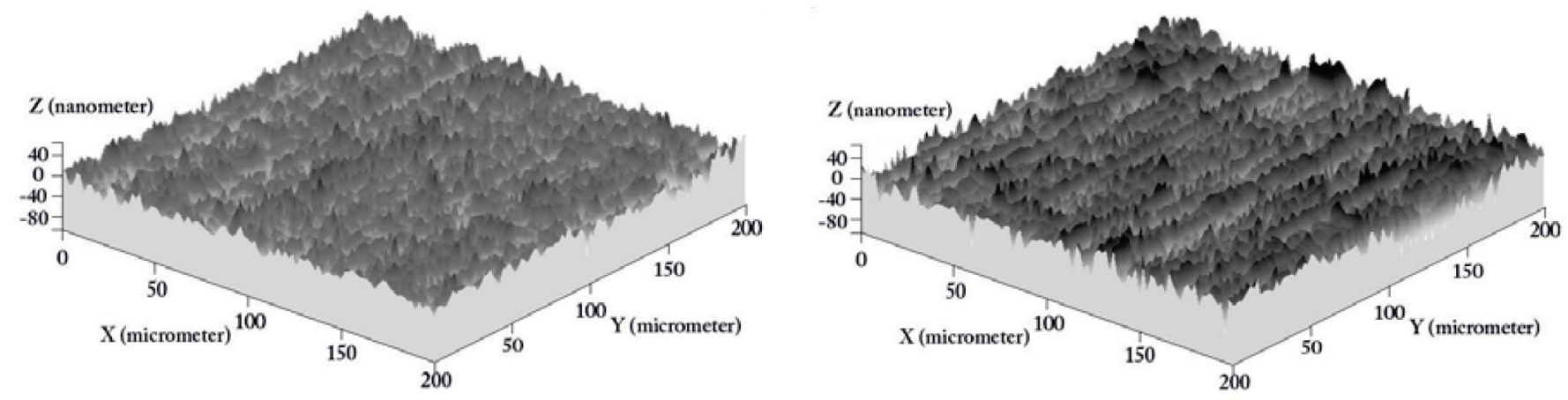

FIG. 7. Three dimensional surface profile of a strained-layer photocathode before (left) and after (right) exposure to atomic deuterium. The rms roughness of the surface is seen to be clearly enhanced by the deuterium. 

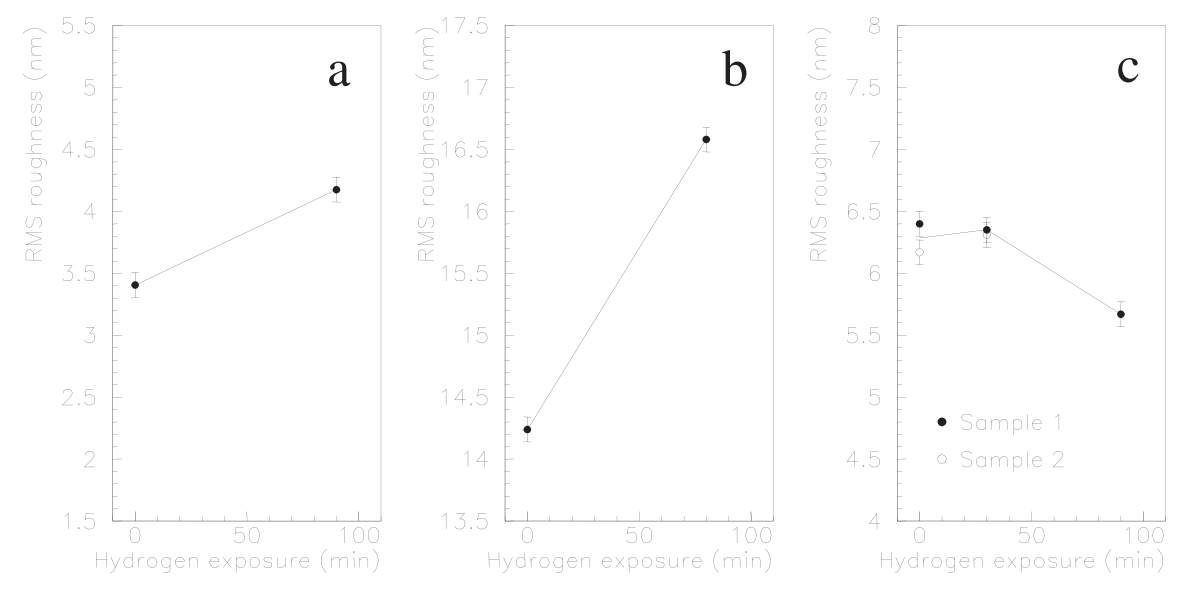

FIG. 8. Rms surface roughness of a bulk (a), a strained layer (b) and two superlattice (c) GaAs photocathodes as a function of exposure to atomic deuterium. Results from two different superlattice wafers (samples 1 and 2) yield comparable results.

time. Results are summarized in Fig. 8. A clear roughening of the surface of both bulk and strained-layer material is detected as a result of atomic deuterium exposure, however no roughening is detected for the superlattice sample. The inconsistent results for the two high-polarization materials and the weak amplitude of the measured roughening suggest that surface effects, namely, arsenic depletion and surface roughening, cannot account for observed experimental results.

It is well known that atomic hydrogen has a high diffusion length in GaAs [24]. Studies indicate that hydrogen can passivate defects within semiconductors and lead to enhancement of material properties such as carrier lifetime and luminescence [25]. However, other studies [26] suggest that high doses of atomic hydrogen lead to degradation of semiconductor material properties. For example, Chang et al [27] report that optical and electrical properties of strained InGaAs/GaAs quantum wells are degraded because high local concentrations of hydrogen within the material lead to the creation of nonradiative recombination centers. High hydrogen concentrations, or clusters, are located primarily at defect sites such as the interface between lattice-mismatched materials. Chang also suggests that hydrogen diffusion and subsequent hydrogen clustering is enhanced by the local strain fields like those present within strained and strained-superlattice structures, even at room temperature. The atomic hydrogen source used throughout this experiment provides a dose of $\sim 10^{20}$ atoms $/ \mathrm{cm}^{2}$ during a 10 min exposure, an amount larger than doses described in Ref. [27]. Therefore it seems plausible to attribute QE degradation observed for both photocathode materials to hydrogen diffusion and the creation of nonradiative recombination centers within the photocathode material. Furthermore, the degradation of the strained GaAs properties via hydrogenation would be enhanced for the superlattice structure, because it contains more numerous interfaces and higher local strains. This would explain why the QE of strained-superlattice GaAs photocathodes decreased more dramatically compared to strained-layer GaAs material. In addition, the hydrogencluster defect states, which are below the Fermi level, would contribute to unpolarized photoemission, therefore lowering the polarization of the extracted beam.

Another possible explanation for hydrogen induced depolarization is lowering of the elastic strain within the heterostructure due to high concentration of hydrogen within the material. It was observed [28] that for high concentrations of atomic hydrogen within GaAs structures with elevated defect densities, hydrogen atoms recombine into hydrogen molecules that accumulate within bubbles of nanometric dimensions. The pressure within these bubbles is so high that it induces microcracks in the semiconductor material. This new type of crystal defect has been observed by means of transmission electron microscopy. Strained heterostructures, such as the ones used in our experiments, contain many crystal defects such as misfit dislocations. It seems possible these defects can initiate the recombination of hydrogen atoms into molecules which gather into bubbles to produce microcracks. The generation of microcracks in turn decreases the strain within the heterostructure and therefore lowers the polarization of the emitted electrons.

\section{CONCLUSIONS}

Measurements indicate vendors provide clean photocathode material and if precautions are taken during preparation procedures, there is no longer a need for atomic hydrogen/deuterium cleaning, a technique that has been employed at Jefferson Lab for years. Excellent quantum efficiency can be obtained from samples as received from the manufacturer [6,8]: 0.15 to $0.3 \% \mathrm{QE}$ at $840 \mathrm{~nm}$ for strained-layer $\mathrm{GaAs}$ and $0.8 \%$ to $1 \% \mathrm{QE}$ from strainedsuperlattice GaAs at $780 \mathrm{~nm}$. Beam polarization is consis- 
tently high from each type of material; $75 \%$ to $80 \%$ for strained-layer GaAs and $80 \%$ to $85 \%$ for strainedsuperlattice GaAs.

Experience at Jefferson Lab indicates that atomic hydrogen/deuterium cleaning can effectively remove contamination from the surface of photocathodes, however it is difficult to know how much cleaning is required. This work illustrates that overexposure to atomic hydrogen/deuterium can dramatically reduce photocathode QE and beam polarization can drop by $\sim 10 \%$ at the band gap. This was confirmed using two different tests stands; one test stand required frequent venting and baking, the other test stand required only one initial bakeout. All results were obtained using an rf-inductive discharge type atomic hydrogen and deuterium source. No attempts were made to explore other types of atomic hydrogen sources.

It seems unlikely that surface effects such as roughening or arsenic depletion can explain observed behavior. The authors speculate that $\mathrm{QE}$ and beam polarization degradation be caused by hydrogen clusters in the photocathode material generating defect sites, or by microcracks created in the semiconductor by high pressure hydrogen filled bubbles. Extensive surface-science techniques must be employed to test these hypotheses.

A recent study done at SLAC reported quantum efficiency degradation from bulk GaAs samples exposed to hydrogen from an rf-inductive discharge dissociator but beam polarization from strained-layer GaAs samples remained fairly stable [29], in contrast to work reported here. As possible explanation, we note that performance of these types of atomic hydrogen sources can vary significantly for small changes to operating conditions, such as absorbed rf power or vacuum pressure near the sample surface. It is possible the JLab and SLAC hydrogen sources provide vastly different amounts of atomic hydrogen at the test sample surface. Moreover, SLAC test samples were exposed to wet chemicals prior to testing, creating different surface conditions and levels of surface cleanliness for each study, therefore impacting the outcome of the hydrogen cleanings.

\section{ACKNOWLEDGMENTS}

This work was supported by the U.S. DOE under Contract No. DE-AC05-84ER401050.

[1] D. T. Pierce et al., Appl. Phys. Lett. 26, 670 (1975).

[2] C.K. Sinclair et al., in SPIN 96: Proceedings of the 12th International Symposium on High-Energy Spin Physics, Amsterdam, The Netherlands, 1996 (World Scientific, Singapore, 1997), p. 739; C.K. Sinclair et al., in Proceedings of the IEEE Particle Accelerator Conference, Vancouver, Canada, 1997 (IEEE, Piscataway, NJ, 1998), p. 2864.
[3] M. Poelker, in LE 98: Proceedings to the Low Energy Polarized Electron Workshop, St. Petersburg, Russia, 1998 (SPES-Lab-Publishing, St. Petersburg, Russia, 1998), p. 105.

[4] K. Aulenbacher et al., in SPIN 2000: Proceedings of the 14th International Spin Physics Symposium, Osaka, Japan, 2000, AIP Conf. Proc. No. 570 (AIP, New York, 2001), p. 949.

[5] B.M. Dunham et al. (NPL Polarized Source Group), University of Illinois, Urbana/Champaign Technical Note No. 90-3; B. Schwartz, F. Ermanis, and M.H. Brastad, J. Electrochem. Soc. 123, 1089 (1976).

[6] Bandwidth Semiconductor, LLC, 25 Sagamore Park Drive, Hudson, NH 03051, http://www.bandwidthsemi.com

[7] T. Maruyama, E.L. Garwin, R. Prepost, and G.H. Zapalac, Phys. Rev. B 46, 4261 (1992).

[8] SVT Associates, Inc., 7620 Executive Drive, Eden Prairie, MN 55344, http://www.svta.com

[9] T. Maruyama et al., Appl. Phys. Lett. 85, 2640 (2004); J.E. Clendenin, in Proceedings to the 10th Workshop on Polarized Sources and Targets, Novosibirsk, Russia, 2003 [Nucl. Instrum. Methods Phys. Res., Sect. A 536, 308 (2005)].

[10] B.M. Dunham, Ph.D. thesis, University of Illinois at Urbana-Champaign, 1993.

[11] M. Baylac, in Proceedings to the Workshop on Polarized Electron Sources and Polarimeters, Danvers, Massachussetts, published with SPIN 2002: 15th International Spin Physics Symposium and Workshop on Polarized Electron Sources and Polarimeters, Upton, NY, 2002, edited by Y.I. Makdisi et al., AIP Conf. Proc. No. 675 (AIP, Melville, NY, 2003), p. 1072.

[12] T. J. Gay and F. B. Dunning, Rev. Sci. Instrum. 63, 1635 (1992).

[13] M. Steigerwald, in SPIN 2000: Proceedings of the 14th International Spin Physics Symposium, Osaka, Japan, 2000 (Ref. [4]), p. 935.

[14] W. W. MacAlpine and R. O. Schildknecht, Proc. IRE 47, 2099 (1958).

[15] J. S. Price and W. Haeberli, Nucl. Instrum. Methods Phys. Res., Sect. A 349, 321 (1994).

[16] M. Poelker, K. P. Coulter, R. J. Holt, C.E. Jones, R. S. Kowalczyk, L. Young, B. Zeidman, and D. K. Toporkov, Phys. Rev. A 50, 2450 (1994).

[17] Beam time is oversubscribed at CEBAF where approximately half of the experiments require a polarized beam. Lower beam polarization would necessitate longer data taking time to maintain the same statistical accuracy and would therefore lower significantly the overall efficiency of the physics program.

[18] M. Yamada and Y. Ide, Jpn. J. Appl. Phys. 33, L671 (1994).

[19] Y. Ide and M. Yamada, J. Vac. Sci. Technol. A 12, 1858 (1994).

[20] E. Petit et al., J. Vac. Sci. Technol. A 10, 2172 (1992).

[21] E. Petit and F. Houzay, J. Vac. Sci. Technol. B 12, 547 (1994).

[22] S. Sugata et al., J. Vac. Sci. Technol. B 6, 1087 (1988).

[23] A.T. Wu, in Proceedings of the 11th Workshop on RFSuperconductivity (SRF2003), Lübeck, Germany 2003 (DESY, Travemunde, 2003). 
[24] S. J. Pearton, J. W. Corbett, and M. Stavola, Hydrogen in Crystalline Semiconductor, Springer Series in Materials Science (Springer-Verlag, Berlin, 1992).

[25] S. J. Pearton et al., Appl. Phys. A 43, 153 (1987); S.J. Pearton et al., in Proceedings of the 15th International Conference on Defects in Semiconductors, Budapest, Hungary, 1988 (Aedermannsdorf, Switzerland,
1989), p. 25; M. Capizzi et al., J. Appl. Phys. 72, 1454 (1992).

[26] N. M. Johnson, F. A. Ponce, R. A. Street and R. J. Nemanich, Phys. Rev. B 35, 4166 (1987).

[27] Y. L. Chang et al., J. Appl. Phys. 75, 3040 (1994).

[28] I. Radu et al., Appl. Phys. Lett. 82, 2413 (2003).

[29] T. Maruyama, Appl. Phys. Lett. 82, 4184 (2003). 